\title{
Treatment of Two Patients with Erythrodermic Psoriasis with Ixekizumab During COVID-19 Pandemic
}

\author{
(1) Melis Gönülal, (1) Didem Didar Balcı
}

Izmir Tepecik Training and Research Hospital, Clinic of Dermatology, Izmir, Turkey

\section{ABSTRACT}

Erythrodermic psoriasis (EP) is the most severe form of psoriasis. Here, we report two cases of EP who achieved Psoriasis Area and Severity Index (PASI) 100 with the treatment of ixekizumab during Coronavirus disease-19 (COVID-19) pandemic. A female and a male patient with EP applied to dermatology outpatient clinic of our hospital. Treatment with standard dose ixekizumab (160 mg sc at week 0 and then $80 \mathrm{mg}$ sc every 2 weeks for 12 weeks) led to PASI 100 response in our first case after only 5 weeks and in our second case after 6 weeks. To date, there have been six patients with EP who reached PASI 100 with ixekizumab treatment, one of them could achieve to this level earliest at week 6 , one of them earliest at week 8 and four of them earliest at week 12, whereas our first case reached PASI 100 at week 5 and our second case reached at week 6 . Our cases were deemed worthy of presentation because our female patient was the case with the fastest response to PASI 100 with ixekizumab, and our two cases were the first reported patients who reached PASI 100 during COVID-19 pandemic. Our patients continue ixekizumab treatment without any side effects and remain self-isolated at home.

Keywords: Psoriasis, Erythrodermic psoriasis, Ixekizumab, COVID-19

\section{Introduction}

Psoriasis, with a worldwide prevalence of $0.5 \%$ to $3 \%$, is a systemic inflammatory disease [1,2]. Erythrodermic psoriasis (EP) is the most severe form of psoriasis [3]. A recent class of biologic agents that has been approved for the treatment of moderate to severe psoriasis is the interleukin (IL)-17 inhibitors, which include secukinumab, brodalumab, and ixekizumab [4]. Ixekizumab is a recombinant, high affinity and humanized monoclonal antibody IgG that inhibits IL-17A [1,3]. There are few case series with EP who became better or treated completely with ixekizumab $[2,5,6]$. Here, we report two cases of EP who achieved Psoriasis Area and Severity Index (PASI) 100 with the treatment of ixekizumab during Coronavirus disease-19 (COVID-19) pandemic.

\section{Case Reports}

\section{Case 1}

A 31-year-old female with EP applied to the dermatology outpatient clinic of our hospital in March 2020. The patient was suffering from plaque-type psoriasis for 18 years. When referring to our clinic, she was affected by an erythrodermic form of psoriasis (Figure 1A, 1B). We learned that she became EP the second time and both first and second EP occurred after her pregnancy. She did not have another disease. PASI was 39.8 at baseline. We began ixekizumab for her on March 23, 2020. We said her that she should be isolated at home because of the COVID-19 outbreak during ixekizumab treatment. Treatment with standard dose ixekizumab (160 mg sc at week 0 and then $80 \mathrm{mg}$ sc every two weeks for 12 weeks) led to PASI 90 response 
after only two weeks. Then, we observed a PASI 100 response to continuing at the patient's fifth-week control (Figure 2A, 2B). No adverse effects developed and until today no relaps have been observed. The patient's consent was obtained for this case study.

\section{Case 2}

A 66-year-old male with EP applied to the dermatology outpatient clinic of our hospital in May 2020. The patient was suffering from plaque-type psoriasis for eight years. When referring to our clinic, he was affected by an erythrodermic form of psoriasis (Figure 3A, 3B). He had primary hypertension. PASI was 54 at baseline. We began ixekizumab for him on May 14, 2020. We said him that he should be isolated at home because of the COVID-19 outbreak during ixekizumab treatment. Treatment with standard dose ixekizumab (160 mg sc at week 0 and then $80 \mathrm{mg}$ sc every two weeks for 12 weeks) led to PASI 100 response after only six weeks (Figure 4A, 4B).
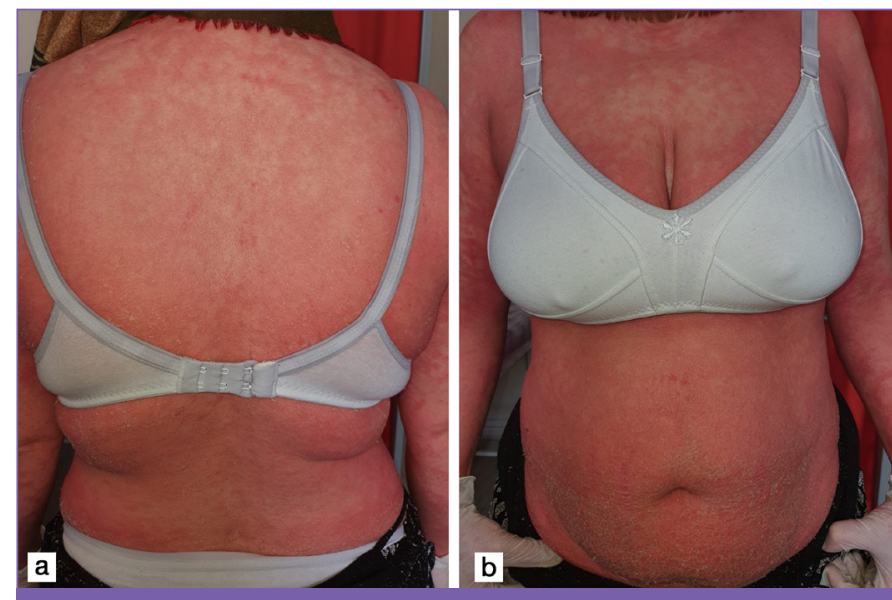

Figure 1. A) Diffuse erythem on the back. B) Diffuse erythem and desquamation in the front of the body

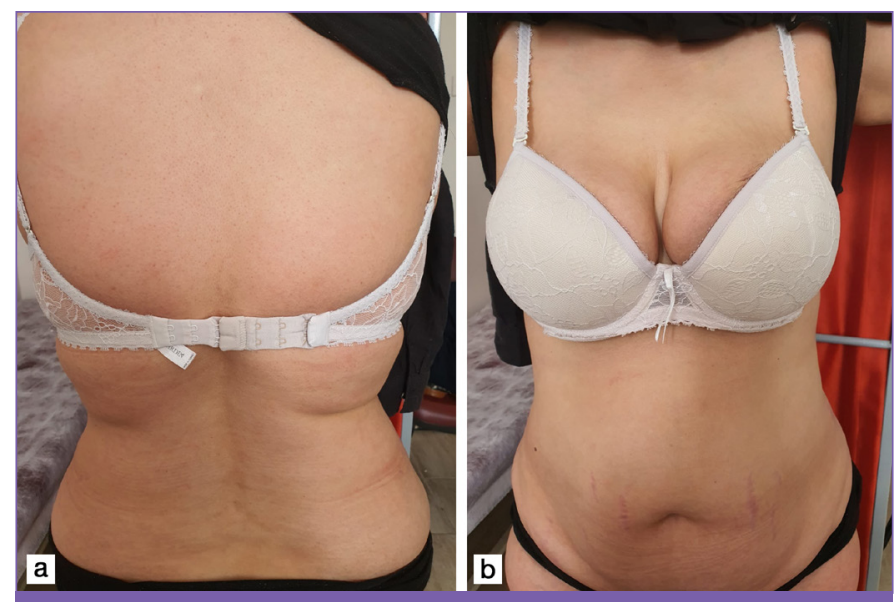

Figure 2. A) Normal skin of the back after healing. B) Normal skin of the front of the body after healing
No adverse effects developed and until today no relaps have been observed. The patient's consent was obtained for this case study.

\section{Discussion}

The first case with COVID-19 was recorded on March 11, 2020, in Turkey. Data on the EP of ixekizumab approved for the treatment of moderate to severe plaque psoriasis have been very limited to date. Megna et al. [3] presented a case with EP who healed with ixekizumab that was able to lead to a complete resolution of the disease after six weeks. In Saeki et al.'s [2] study, 100.0\% of the EP patients achieved PASI 75, 62.5\% (5/8) achieved PASI 90 and 25.0\% (2/8) achieved PASI 100 at week 12 and $100.0 \%$ of the patients maintained PASI 75, 87.5\% (7/8) patients achieved PASI 90 and $12.5 \%$ (1/8) patients achieved PASI 100 at week 24. In another study of Saeki et al. [7], it was presented that for eight patients with EP, global improvement scores indicated that all patients either
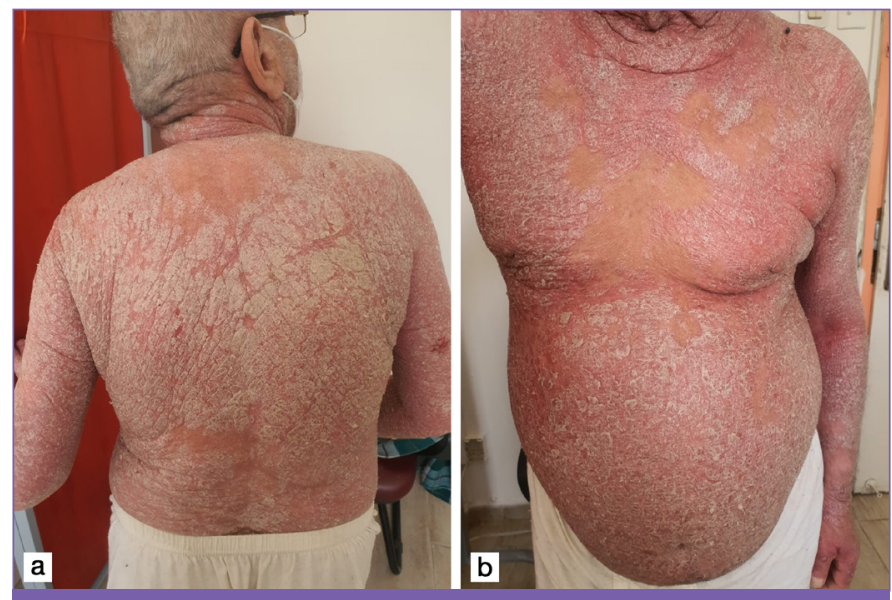

Figure 3. A) Diffuse erythem, desquamation and squams on the back. B) Diffuse erythem, desquamation and squams on the front of the body

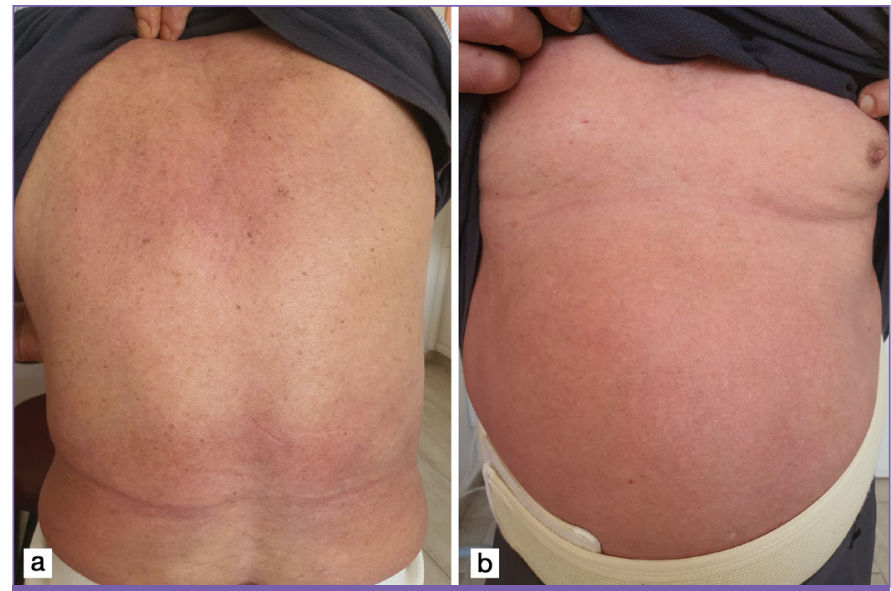

Figure 4. A) Normal skin of the back after healing. B) Normal skin of the front of the body after healing 
resolved or improved by week 52 of the ixekizumab treatment. Improvements in PASI were observed at 12 weeks and maintained for the 52-week treatment period. All patients with EP responded (improved or resolved) to ixekizumab treatment. At week 52, six of the eight patients with EP reached PASI 90 response. In Carrasquillo et al.'s [5] study, eight patients were treated with ixekizumab as part of an open-label study. By week 12, all patients achieved PASI 75, 5/8 achieved PASI 90, and 2/8 achieved PASI 100. By week 24, $100 \%$ of the patients reached PASI 75, 7/8 reached PASI 90 , and 1/8 reached PASI 100. After 52 weeks of follow-up, ixekizumab achieved significant improvement. According to this research, ixekizumab can be considered first-line treatments for EP. In Lo and Tsai's [6] study, at week 12, seven (78\%) patients (total nine patients) achieved PASI 50; of these, four (44\%) patients one (11\%) patient achieved PASI 75 (75\% reduction in PASI) and PASI 90 (90\% reduction in PASI), respectively. The patient who achieved PASI 90 actually achieved PASI 100 at weeks 8 and 12. Okubo et al. [8] presented in their study that all eight patients had early and sustained improvement in PASI scores with ixekizumab treatment. In their research, the mean PASI score was 42.8 at baseline, 3.0 at week 52 , and 5.0 at week 244 . None of the patients with EP reached PASI 100. It was not explained in detail that how many patients reached PASI 50-75-90, but according to mean PASI score at week 52, there were few patients who reached PASI 90 response.

According to some studies, biologics were used safely during the COVID-19 pandemic. In Gisondi et al.'s [9] study, their findings showed that there was not a significant number of hospitalizations or deaths from COVID-19 (a multicentric study, 5206 cases with chronic plaque psoriasis being treated with biologic therapy). In another study conducted by Gisondi et al. [10], it was observed that among 980 patients with chronic plaque psoriasis on biologics, there were no cases of hospitalization or death. Additionally, a case from Italy who was 55 years old had contacted a COVID positive patient on his induction stage with ixekizumab, and then, he was tested for Severe acute respiratory syndrome-Coronavirus-2 (SARS-CoV-2) resulted positive. He did not provide his doctors with this information and continued to use ixekizumab. His control test for SARS-CoV-2 resulted in negative, and in this process, he confirmed never having suffered from cough, dyspnea, anosmia, ageusia, myalgia or any other symptom of the infection. Interestingly, the IL-23/IL-17 axis does not seem to be pivotal in an effective immune response [11]. On the contrary, observations carried on both Coronavirus, and nonCoronavirus pneumonia patients show that an aberrant Th17 polarization may correlate with a worse outcome. Based on these observations, a clinical trial investigating the use of ixekizumab associated with antiviral therapy is currently ongoing in China as a possible treatment for COVID-19 infection. During COVID-19, inflammatory cytokines play a double role. Firstly, they stimulate effective immune response activation and then can mediate the development of exaggerated systemic inflammation. This cytokine storm is not effective on the pathogen of COVID-19 [12]. The outcome of data from currently available literature suggests that IL-23/IL-17 axis inhibition may not be detrimental in the setting of COVID-19 infection. Further data are needed to support this hypothesis.

To date, there have been six patients with EP who reached PASI 100 with ixekizumab treatment, one of them could achieve to this level earliest at week 6 , one of them earliest at week 8 and four of them earliest at week 12, whereas our first case reached PASI 100 at week 5 and our second case reached at week 6 . Our cases were deemed worthy of presentation because our female patient was the case with the fastest response to PASI 100 with ixekizumab, and our two cases were the first reported patients who reached PASI 100 during COVID-19 pandemic. Our patients continue ixekizumab treatment without any side effects and remain self-isolated at home.

\section{Ethics}

Informed Consent: Consent form was filled out by all participants.

Peer-review: Internally peer-reviewed.

\section{Authorship Contributions}

Surgical and Medical Practices: M.G., D.D.B., Concept: M.G., D.D.B., Design: M.G., D.D.B., Data Collection or Processing: M.G., D.D.B., Analysis or Interpretation: M.G., D.D.B., Literature Search: M.G., D.D.B., Writing: M.G., D.D.B.

Conflict of Interest: No conflict of interest was declared by the authors.

Financial Disclosure: The authors declared that this study received no financial support.

\section{References}

1. Gulliver W, Penney M, Power R, Gulliver S, Montmayeur S, Burge R. Moderateto-severe plaque psoriasis patients treated with ixekizumab: early real-world outcomes and adverse events. J Dermatolog Treat 2020:1-7.

2. Saeki H, Nakagawa H, Ishii T, Morisaki Y, Aoki T, Berclaz PY, Heffernan M. Efficacy and safety of open-label ixekizumab treatment in Japanese patients with moderate-to-severe plaque psoriasis, erythrodermic psoriasis and generalized pustular psoriasis. J Eur Acad Dermatol Venereol 2015;29:11481155.

3. Megna M, Gallo L, Balato N, Balato A. A case of erythrodermic psoriasis successfully treated with ixekizumab. Dermatol Ther 2019;32:e12825.

4. Martin DA, Towne JE, Kricorian G, Klekotka P, Gudjonsson JE, Krueger JG, Russell CB. The emerging role of $\mathrm{IL}-17$ in the pathogenesis of psoriasis: preclinical and clinical findings. J Invest Dermatol 2013;133:17-26.

5. Carrasquillo OY, Pabón-Cartagena G, Falto-Aizpurua LA, Santiago-Vázquez M, Cancel-Artau KJ, Arias-Berrios G, Martín-García RF. Treatment of erythrodermic psoriasis with biologics: A systematic review. J Am Acad Dermatol 2020;83:151-158. 
6. Lo Y, Tsai TF. Clinical experience of ixekizumab in the treatment of patients with history of chronic erythrodermic psoriasis who failed secukinumab: a case series. Br J Dermatol 2019;181:1106-1107.

7. Saeki H, Nakagawa H, Nakajo K, Ishii T, Morisaki Y, Aoki T, Cameron GS, Osuntokun 00; Japanese Ixekizumab Study Group. Efficacy and safety of ixekizumab treatment for Japanese patients with moderate to severe plaque psoriasis, erythrodermic psoriasis and generalized pustular psoriasis: Results from a 52-week, open-label, phase 3 study (UNCOVER-J). J Dermatol 2017;44:355-362.

8. Okubo Y, Mabuchi T, Iwatsuki K, Elmaraghy H, Torisu-Itakura H, Morisaki Y, Nakajo K. Long-term efficacy and safety of ixekizumab in Japanese patients with erythrodermic or generalized pustular psoriasis: subgroup analyses of an open-label, phase 3 study (UNCOVER-J). J Eur Acad Dermatol Venereol 2019;33:325-332.

9. Gisondi P, Facheris P, Dapavo P, Piaserico S, Conti A, Naldi L, Cazzaniga S, Malagoli P, Costanzo A. The impact of the COVID-19 pandemic on patients with chronic plaque psoriasis being treated with biological therapy: the Northern Italy experience. Br J Dermatol 2020;183:373-374.

10. Gisondi P, Zaza G, Del Giglio M, Rossi M, lacono V, Girolomoni G. Risk of hospitalization and death from COVID-19 infection in patients with chronic plaque psoriasis receiving a biologic treatment and renal transplant recipients in maintenance immunosuppressive treatment. J Am Acad Dermatol 2020;83:285-287.

11. Messina F, Piaserico S. SARS-CoV-2 infection in a psoriatic patient treated with IL-23 inhibitor. J Eur Acad Dermatol Venereol 2020;34:e254-e255.

12. Liu P, Huang Z, Yin M, Liu C, Chen X, Pan P, Kuang Y. Safety and efficacy of ixekizumab and antiviral treatment for patients with COVID-19: a structured summary of a study protocol for a pilot randomized controlled trial. Trials 2020;21:999. 